\section{VOCAL CORD \\ MEDIALIZATION FOR \\ UNILATERAL PARALYSIS \\ ASSOCIATED WITH \\ INTRATHORACIC \\ MALIGNANCIES}

Patients with unilateral vocal cord paralysis from intrathoracic malignancies may have significant dysfunctions of speech, swallowing, ventilation, and effective coughing as a result of inadequate compensation of the nonparalyzed cord. In patients with already compromised pulmonary function, aspiration can be a life-threatening event. Sixty-three patients with intrathoracic malignancies required surgical correction of vocal cord paralysis. Primary pathology included lung cancer (49), esophageal cancer (nine), and miscellaneous tumors (five). Symptoms included hoarseness (62), dyspnea (21), aspiration (26), weight loss (19), dysphagia (14), and pneumonia (14). The surgical procedures included medial displacement of the vocal cord with silicone elastomer (48), temporary Gelfoam injection (seven), and Teflon (polytetrafluoroethylene) injection (eight) to move the affected cord to a medial position. In 11 patients, the operation was performed in the acute postoperative setting to improve pulmonary toilet. Symptomatic improvement was noted in the following proportions of affected patients: hoarseness, $92 \%$; dyspnea, $90 \%$; dysphagia, $93 \%$; aspiration, 92\%; pneumonia, 93\%; and weight loss, 47\%. Overall success rate of the intervention was 57 of 63 patients $(90 \%)$. All 11 patients treated in the acute setting had immediate improvement. A variety of complications occurred in $17 \%$ of patients. Surgical management of vocal cord paralysis in patients with intrathoracic malignancies prevents life-threatening pulmonary complications in the acute postoperative setting. In chronic situations, it provides patients with improved speech, swallowing, and pulmonary function, resulting in improved quality of life, even for patients not cured of their disease. (J THORAC Cardiovasc SuRg 1996;111:334-41)

Dennis H. Kraus, MD (by invitation), ${ }^{a}$ Muhammed K. Ali, MD

(by invitation), ${ }^{\mathrm{b}}$ Robert J. Ginsberg, MD, ${ }^{\mathrm{c}}$ Christopher J. Hughes, MD

(by invitation), ${ }^{a}$ Robert F. Orlikoff, $\mathrm{PhD}$ (by invitation), ${ }^{\mathrm{a}}$

Valerie W. Rusch, MD, ${ }^{\mathrm{c}}$ Michael E. Burt, MD, ${ }^{\mathrm{c}}$

Patricia M. McCormack, MD, ${ }^{c}$ and Manjit S. Bains, MD, ${ }^{c}$

New York and Flushing, N.Y.
Intrathoracic malignant neoplasms are associated with a variety of local complications caused by the primary tumor, the mediastinal lymph nodes, or the

From the Speech, Hearing, and Rehabilitation Center, Head and Neck Service, ${ }^{a}$ and the Thoracic Surgery Service, ${ }^{c}$ Department of Surgery, Memorial Sloan-Kettering Cancer Center, New York, and The Department of Surgery, Flushing Hospital Medical Center, ${ }^{\mathrm{b}}$ Flushing, N.Y.

Read at the Seventy-fifth Annual Meeting of The American Association for Thoracic Surgery, Boston, Mass., April 23-26, 1995.

Address for reprints: Dennis H. Kraus, MD, Box 285, 1275 York Ave., Memorial Sloan-Kettering Cancer Center, New York, NY 10021.

Copyright (C) 1996 by Mosby-Year Book, Inc.

$0022-5223 / 96 \$ 5.00+0 \quad \mathbf{1 2 / 6 / 6 9 6 6 4}$ surgical treatment of the disease. Carcinomas of the lung and esophagus, which account for the majority of these tumors, commonly involve intrathoracic neurovascular structures. One compromising sequela of this involvement is unilateral vocal cord paralysis (UVCP) as a result of direct tumor or lymphatic invasion of the vagus or recurrent laryngeal nerve or tumor location, necessitating resection or manipulation of the intrathoracic segment of the nerve.

The ability of patients to tolerate UVCP is extremely variable. Temporary neuropraxia may last a few weeks or as long as 6 to 9 months. Partial palsies are often tolerated because of compensation by the contralateral vocal cord. Permanent paralysis is tolerated to different degrees, depending on the final 
resting position of the vocal cord and the ability of the contralateral cord to compensate. Compensatory mechanisms are less able in older patients, however, to prevent hoarseness and aspiration from becoming detrimental. ${ }^{1}$ Even minimal aspiration may be debilitating to patients whose pulmonary reserve has been diminished by years of smoking, chronic obstructive pulmonary disease, and other cardiopulmonary comorbidities. Lack of an effective cough and loss of modulation of the expiratory phase of respiration further increase the risk of pulmonary complications. Patients with intrathoracic malignancy, who are frequently compromised by age and respiratory disease, therefore tend to be less tolerant of UVCP.

Movement of the vocal cords to a medial position ("medialization") in patients with UVCP is a means of improving speech and swallowing function, and of diminishing pulmonary dysfunction. Limited data exist regarding the impact of this intervention in patients with an intrathoracic malignancy. Medialization can be achieved directly by endolaryngeal injection or by external means with transcutaneous insertion of a surgically positioned implant. We review our experience with these procedures in 63 consecutive patients with intrathoracic malignancy complicated by UVCP.

\section{Patients and methods}

From July 1, 1991, through December 31, 1994, a total of 63 patients with intrathoracic malignancies underwent vocal cord medialization for UVCP at Memorial SloanKettering Cancer Center. A retrospective chart review was performed to document the type and stage of the malignancy treated, associated symptoms, treatment, cause of UVCP and extent of compensation, indications for and the type of medialization, and outcome of the medialization.

All procedures were performed with a combination of topical, local, and intravenous sedative anesthesia. Injection of Gelfoam (Upjohn, Kalamazoo, Mich.) or Teflon (polytetrafluoroethylene) (Mentor, Norwell, Mass.) was performed with the patient in a flexed "sniffing" position. An anterior commissure laryngoscope was used to visualize the larynx. The needle of the injection gun was placed deep within the substance of the vocalis muscle lateral and anterior to the arytenoid. Under direct vision, material was injected to cause the medial displacement of the vocal cord (Figs. 1 and 2).

Silicone elastomer medialization, the surgical implantation of a handcrafted silicone elastomer implant by the modified technique of Isshiki and coworkers, ${ }^{2}$ was performed with the patient in a supine position. A natural skin crease over the affected midthyroid laminae was used. Flaps were elevated in a subplatysmal plane. The strap muscles were identified in the midline. Strap muscles were

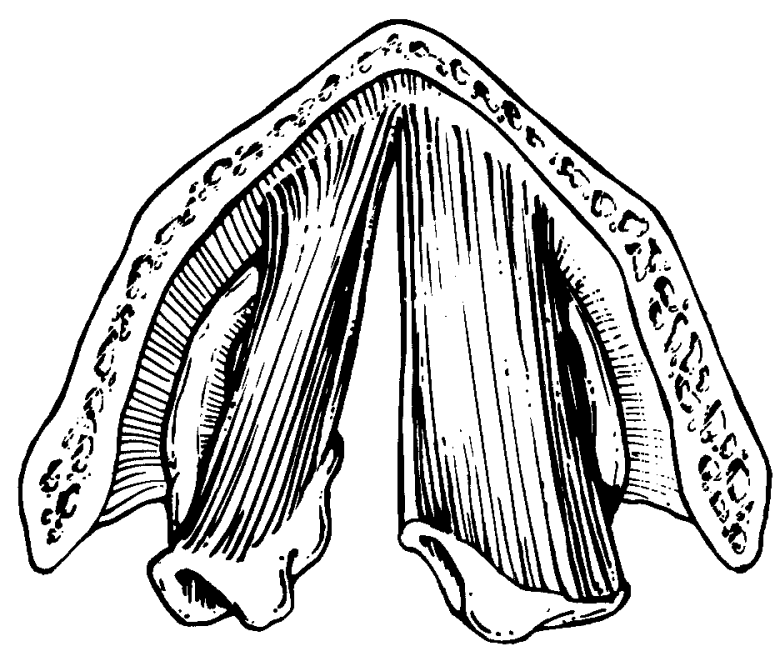

Fig. 1. Axial section of the larynx with left UVCP on phonation with poor compensation.

elevated free of the affected thyroid lamina. A thyrotomy window was created with an otologic bur approximately 7 $\mathrm{mm}$ from the midline. The window typically measured $6 \times$ $10 \mathrm{~mm}$ in a rectangular fashion. The upper border of the window was in the midpoint of the superior-inferior dimension. An otologic bur was used to remove the cartilaginous window. Care was taken to preserve the inner perichondrium to allow creation of an inner pocket. The implant was designed in a lock-and-key configuration (Fig. 3). The length of the implant measured 10 to $12 \mathrm{~mm}$, the anterior height was 1.5 to $2.5 \mathrm{~mm}$, and the posterior height was 3.5 to $6.0 \mathrm{~mm}$. Additional fixation was not required because of the lock-and-key configuration of the implant. The wound was closed in multiple layers with absorbable sutures.

The patients were counseled regarding potential fluctuations in voice during the first 6 weeks, related to edema at the operative site. Improvement in dysphagia was identified immediately. A foreign-body sensation was present for 1 to 2 weeks. Operative timing was defined as "acute" when the operation was performed within 2 weeks of operative disruption of the recurrent laryngeal nerve, "elective" when the procedure was performed 2 weeks to 6 months after manifestation of paralysis, and "chronic" when it was performed 6 months after development of UVCP.

Evaluation of preoperative speech, swallowing, and pulmonary disability was based on patient and surgeon (D. H. K.) assessment. Physical examination focused on the separation (in millimeters) of the vocal cords at the posterior commissure on phonation. Postoperative assessment included the patient's and physician's assessment of speech, swallowing, and pulmonary function, measurement of the gap between the vocal cords at the posterior commissure, and the use of chest radiographs and recorded weights to determine improvement. Patients in whom all symptoms were improved were considered to have a successful result. All patients included in this series were followed up for a minimum of 3 months. 

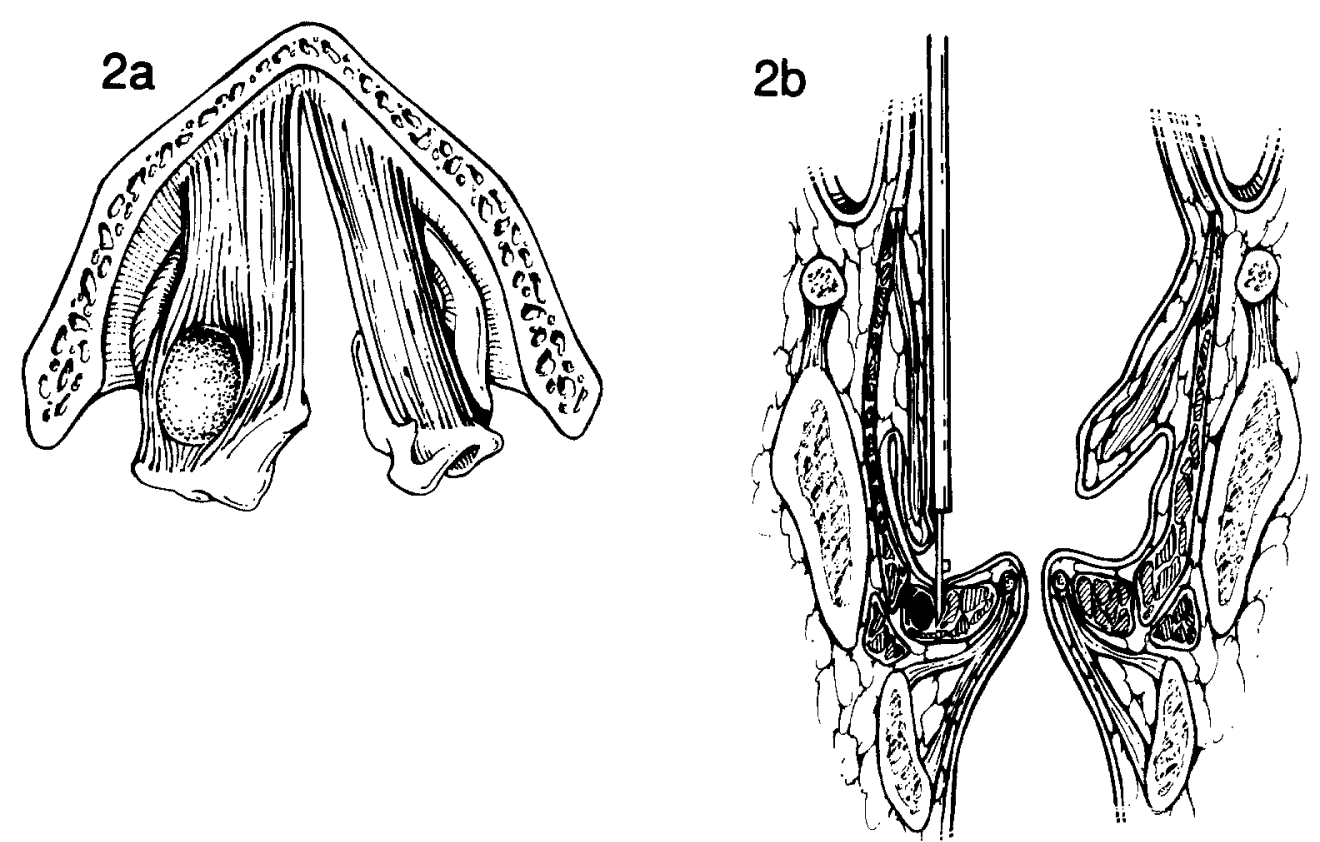

Fig. 2. a, Axial section of Teflon injection with medialization of left true vocal cord. b, Coronal section of Teflon injection with placement of Teflon deep within the substance of the thyroarytenoid muscle.

\section{Results}

There were 51 men and 12 women, with a median age of 64 years (range 36 to 87 years). Carcinoma of the lung was the most common underlying malignancy, occurring in 49 patients; carcinoma of the esophagus occurred in nine, and other miscellaneous tumor types were seen in five. The left vocal cord was paralyzed in 51 patients, the right in 12 . UVCP was caused by the extent of the primary tumor in 35 patients and by intentional resection or manipulation of the vagus or recurrent laryngeal nerve in 28. Symptoms before medialization included; hoarseness (62), aspiration (26), dyspnea (21), weight loss (19), dysphagia (16), and pneumonia (14; Table I). The distance of the gap at the posterior one third of the vocal cords was estimated to be $1.0 \mathrm{~mm}$ in 7 patients, $1.5 \mathrm{~mm}$ in $33,2.0 \mathrm{~mm}$ in $18,2.5 \mathrm{~mm}$ in four, and $3.0 \mathrm{~mm}$ in one (Table II).

Medialization for UVCP was performed in the acute postoperative setting for 11 patients, electively for 28 patients, and in the chronic setting for 24 patients. The primary indications for medialization in the acute setting were inability to generate an adequate cough (six), aspiration (four), and dysphagia (one). In the elective and chronic settings, indications included hoarseness (24), aspiration
(20), dysphagia (eight), and pneumonia (one). Medialization was performed on an outpatient basis for two patients, as an intercurrent procedure during hospital admission in 23 cases, and during a separate admission for 38 patients. Seven patients underwent temporary medialization with endoscopic injection of Gelfoam into the flaccid cord; in one case, this procedure was performed twice. Eight patients underwent permanent medialization with endoscopic Teflon injection (Figs. 1 and 2). Forty-eight patients underwent permanent surgical implantation of a handcrafted silicone elastomer implant by the modified technique of Isshiki and associates ${ }^{2}$ (Fig. 3); for 43 this was a primary procedure, for four it was performed after endoscopic medialization with Gelfoam, and for one it was performed after Teflon injection. The median operative time for endoscopic injection was 25 minutes; that for silicone elastomer medialization was 45 minutes. Blood loss was less than $30 \mathrm{ml}$ for all procedures.

After operation, most patients had no discernable separation of the medialized and mobile cords on phonation (Table I). There was no residual gap after medialization in 35 patients, there was a gap of 0.5 $\mathrm{mm}$ in 23 patients, and there was a gap of $1.0 \mathrm{~mm}$ in five patients. All patients had a reduction in the gap 
Volume 111, Number 2

Table I. Vocal cord gap in patients undergoing medialization

\begin{tabular}{ccc}
\hline Glottic gap $(\mathrm{mm})$ & Preoperative & Postoperative \\
\hline 0 & 0 & 35 \\
0.5 & 0 & 23 \\
1.0 & 7 & 5 \\
1.5 & 33 & 0 \\
2.0 & 18 & 0 \\
2.5 & 4 & 0 \\
3.0 & 1 & 0 \\
\hline
\end{tabular}

Table II. Symptomatic improvement in patients undergoing medialization

\begin{tabular}{lccc}
\hline \multicolumn{1}{c}{ Symptoms } & No. of patients & No. improved & \% improved \\
\hline Hoarseness & 62 & 57 & 92 \\
Aspiration & 26 & 24 & 92 \\
Dyspnea & 21 & 19 & 90 \\
Weight loss & 19 & 9 & 47 \\
Dysphagia & 15 & 14 & 93 \\
Pneumonia & 14 & 13 & 93 \\
\hline
\end{tabular}

between the posterior one third of the vocal cords on phonation. The decrease in the gap size was 0.5 $\mathrm{mm}$ in three patients, $1.0 \mathrm{~mm}$ in $19,1.5 \mathrm{~mm}$ in $25,2.0$ $\mathrm{mm}$ in 14 , and $2.5 \mathrm{~mm}$ in two.

Vocal cord medialization was successful in 57 of 63 patients $(90 \%)$. All patients treated in the acute setting had symptomatic improvement (Table II). Only $47 \%$ of patients with weight loss had a subsequent increase in weight; all of the subsequent weight loss was caused by progression of disease, however, rather than inability to swallow. Successful results were obtained in $88 \%$ of the patients treated with Teflon injection (7/8) and $94 \%$ of those treated with silicone elastomer medialization (45/48). There was no long-term deterioration in results.

Follow-up has ranged from 1 to 30 months (median 4 months). Currently, 19 patients are alive and free of disease (follow-up 3 to 30 months, median 6 months), 12 are alive with disease (range 3 to 15 months, median 4 months), and 32 are dead of their disease (range 1 to 20 months, median 4 months). Among the 31 patients currently alive, only two have unsuccessful long-term results, both as a consequence of complications.

Length of hospitalization after medialization ranged from 0 to 122 days (median 1 day). Of 40 patients undergoing elective admission for medialization, two patients were discharged on the day of operation, 35 were discharged 1 day after operation, and three were hospitalized longer than 1 day $(2,4$,
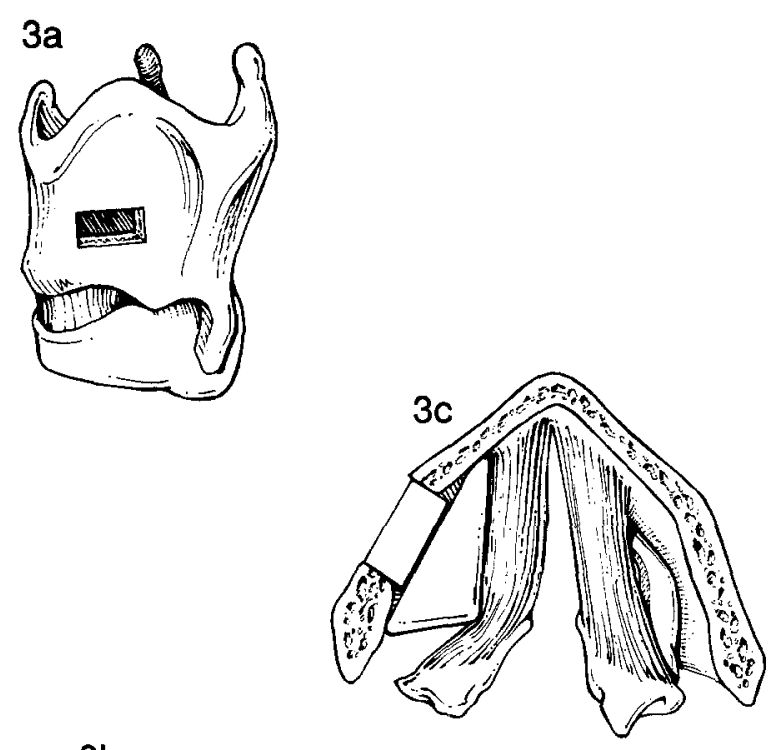

$3 b$

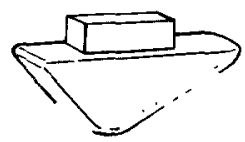

Fig. 3. a, Thyrotomy window with creation of pocket for placement of silicone elastomer implant. $\mathbf{b}$, Handcrafted silicone elastomer implant. c, Axial section of implant in situ with medialization and compensation of the paralyzed left true vocal cord.

and 10 days) because of confounding factors not related to medialization. Among 23 patients undergoing medialization as part of a previous admission, one was discharged on the day of medialization, four were discharged 1 day after medialization, nine patients were hospitalized for between 4 and 9 days, eight patients were hospitalized for between 12 and 18 days, and one patient was hospitalized for 122 days. Prolonged hospitalization was a consequence of the patient's underlying malignancy and not related to medialization.

Complications occurred in 11 patients $(17 \%)$. These were usually minor and included transient airway compromise (three), tooth fracture (one), hematoma (one), foreign-body sensation (two), and transient laryngeal edema (four). Two major complications occurred in this series. The first was a spontaneous extrusion of the implant, associated with paroxsmal coughing, 4 months after successful medialization. The second complication was airway obstruction in a patient undergoing a second Gelfoam injection. This patient's first procedure had been successful. The patient had significant im- 
provement in her voice after the second Gelfoam injection but remained hospitalized because of persistent nausea and vomiting. Sudden airway obstruction developed on the first postoperative day, necessitating emergency tracheostomy, and the patient remained hospitalized for 10 days after the tracheostomy. She refused permanent silicone elastomer medialization and her voice allows adequate communication.

\section{Discussion}

There are limited data on the impact of UVCP in patients with intrathoracic malignancies and on the impact of surgical rehabilitation. This study was performed to determine the effect of surgical medialization in a selected population. UVCP is a common sequela of intrathoracic malignancys. In a group of eight pooled series of UVCPs consisting of 1019 patients, $36 \%$ of cases were caused by neoplasms, with more than half of these resulting from lung cancer. ${ }^{3}$ The ability to compensate for UVCP is variable. Woo and colleagues ${ }^{1}$ associated more severe vocal dysfunction with older patients, male patients, a diagnosis of cancer, and weight loss. These authors were unable to correlate any of those factors with the presence of aspiration. The inability to compensate for aspiration is a result of the degree of vocal cord atrophy and loss of pulmonary support. In patients with UVCP caused by esophageal cancer surgery, Hirano and colleagues ${ }^{4}$ found that $53 \%$ aspirated with swallowing and $45 \%$ had aspiration pneumonia. Tracheostomy was necessary in $25 \%$ for the management of persistent aspiration.

All of our patients had severe hoarseness, which impaired communication. We did not perform objective preoperative and postoperative voice testing, but clinical findings on indirect laryngoscopy are known to correlate with decibel output, phonation time, and mean flow rate after vocal cord medialization. ${ }^{1}$ Gray and associates ${ }^{5}$ compared voice in patients undergoing silicone elastomer medialization to voice in normal control subjects. Voice was comparable with respect to pitch, intonation, and loudness, with abnormal values obtained for strained, breathy, hoarse, and harsh items. The frequency and intensity ranges were decreased for the medialization group. In this same population, in response to a quality of life questionnaire, $92 \%$ noted improvement in voice and $93 \%$ noted voice did not interfere with family interactions, but $25 \%$ stated that persistent voice change necessitated adjustments in employment. In an analysis of long- term effect on voice in patients undergoing the Isshiki procedure, Leder and Sasaki ${ }^{6}$ identified high fundamental frequency, decreased habitual voice intensity, and prolonged maximum phonation time.

Dysphagia or clinically evident aspiration was present in $56 \%$ of our patients. There have been no reports objectively documenting improvement in swallowing after medialization. Netterville and coworkers ${ }^{7}$ have performed primary medializations in patients undergoing lateral skull-base resection with sacrifice of both the superior laryngeal and the recurrent laryngeal nerves. Usually, these patients require tracheostomy and a gastric feeding tube for management of aspiration; this was avoided after primary medialization. Koufman ${ }^{8}$ documented improved swallowing in all patients undergoing medialization, with mild aspiration in one of 11 patients. Aspiration resolved in 13 of 17 patients who underwent some form of medialization for UVCP associated with esophageal cancer surgery. ${ }^{4}$ One patient believed to have aspiration in the series reported here underwent modified barium swallow examination before and after silicone elastomer medialization. The patient had undergone an unsuccessful Teflon injection at another institution and had a gastrostomy for feeding. The postmedialization study documents resolution of aspiration, and the patient was able to discontinue enteral feedings and gain weight eating normally.

Limited morbidity and no mortality were seen in this series. In a series of 60 patients, Tucker and associates $^{9}$ reported a $10 \%$ major complication rate consisting of hematoma; all patients required temporary tracheostomy. In addition, $7 \%$ had extrusion of the implant. Koufman ${ }^{8}$ reported $18 \%$ incidence of overall complications in 11 patients undergoing silicone elastomer medialization. Thes complications included wound infection, implant extrusion, pharyngocutaneous fistula, and airway obstruction. Gardner, Shaari, and Parnes ${ }^{10}$ treated 56 patients with UVCP with a variety of Gelfoam, Teflon, and silicone elastomer medialization techniques. In that series, two patients undergoing Teflon infection had airway obstruction necessitating tracheostomy. The reduced morbidity in our series may be the result of refinements in silicone elastomer medialization technique that have limited local wound complications.

Life expectancy for these patients with advancedstage disease is often short. In our series, because of progressive disease, overall survival has been poor (31 of 63 patients are currently alive). There was a 22\% 5-year accrued survival among patients with 
vocal cord paralysis after esophageal cancer operation. ${ }^{4}$ Gardner, Shaari, and Parnes ${ }^{10}$ noted that patients with UVCP caused by lung cancer had the worst prognosis of all patients, with only $20 \%$ survival 1 year after medialization. The improved quality of life and the ability to speak and eat appears to justify this intervention, however, even in palliative situations.

The choice of technique should be influenced by assessing life expectancy and possible spontaneous return of function. Laryngeal electromyography can distinguish between permanent injury, in which reinnervation is unlikely, and temporary injury, with a good prognosis for return of laryngeal function. For patients with a potentially intact laryngeal innervation, a temporary, reversible procedure such as Gelfoam or silicone elastomer medialization should be employed. For patients in whom return of laryngeal function is not anticipated, silicone elastomer medialization and Teflon injection have been used. Teflon has the disadvantages of not being reversible, lacking the opportunity for fine adjustments, and carrying the potential for long-term formation of Teflon granulomas. Endoscopic Teflon injection requires slightly less time to perform than silicone elastomer implantation. Silicone elastomer implantation requires an external incision, as well as increased operating time; however, there is no local inflammatory response. ${ }^{11}$ Several authors have reported the expeditious performance of silicone elastomer medialization, $2,5,7,9,12$ and the average operating time in our series was 45 minutes. In patients with deficient superior laryngeal nerve innervation, arytenoid adduction can be a useful adjunct to or substitute for silicone elastomer medialization. ${ }^{7}$ This procedure addresses the extreme lateralization or "cadaveric" position of the paralyzed vocal cord in patients with paralysis of both superior and recurrent laryngeal nerves. Because all patients reported on in our series have intact superior laryngeal innervation, we have not found this procedure to be necessary.

A feature of our experience has been an increasing reliance on the use of silicone elastomer medialization because of consistency of results, durability, and technical versatility, including simple removal if cord mobility recovers. We believe that its use is justified in even short-term palliative situations because of its impact on quality of life and its lack of morbidity. In acute postoperative situations, we operated as early as 2 days after thoracotomy, with a time-limiting factor being the allowance for reduction in postintubation laryngeal edema. A number of patients (11) have subsequently required general anesthesia and intubation after silicone elastomer medialization as a result of underlying malignancy, with no reported complications and with maintenance of voice and swallowing.

We thank the attending staff from the Thoracic Radiation Oncology and Medical Oncology services. We also thank Ms. Christine A. Schaar and Ms. Hilary Cathcart for their expert assistance in medical illustration and manuscript preparation.

\section{REFERENCES}

1. Woo P, Colton R, Brewer D, Casper J. Functional staging for vocal cord paralysis. Otolaryngol Head Neck Surg 1991;105:440-8.

2. Isshiki $\mathrm{N}$, Morita $\mathrm{H}$, Okamura $\mathrm{H}$, Hiramoto $\mathrm{M}$. Thyroplasty as a new phonosurgical technique. Acta Otolaryngol 1974;78:451-7.

3. Terris DJ, Arnstein DP, Nguyen HH. Contemporary evaluation of unilateral vocal cord paralysis. Otolaryngol Head Neck Surg 1992;107:84-90.

4. Hirano M, Fujita M, Tanaka S, Fujita H. Vocal cord paralysis caused by esophageal cancer surgery. Ann Otol Rhinol Laryngol 1993;102:182-5.

5. Gray SD, Barkmeier J, Jones D, Titze I, Druker D. Vocal evaluation of thyroplastic surgery in the treatment of unilateral vocal cord paralysis. Laryngoscope 1992;102:415-21.

6. Leder SB, Sasaki CT. Long-term changes in vocal quality following Isshiki thyroplasty type I. Laryngoscope 1994;104:275-7.

7. Netterville JL, Stone JE, Civantos FJ, Luken ES, Ossoff RH. Silastic medialization and arytenoid adduction: the Vanderbilt experience. Ann Otol Rhinol Laryngol 1993;102:413-24.

8. Koufman JA. Laryngoplasty for vocal cord medialization: an alternative to teflon. Laryngoscope 1986;96: 726-31.

9. Tucker HM, Wanamaker J, Trott M, Hicks D. Complications of laryngeal framework surgery (phonosurgery). Laryngoscope 1993;103:525-8.

10. Gardner GM, Shaari CM, Parnes SM. Long-term morbidity and mortality in patients undergoing surgery for unilateral vocal cord paralysis. Laryngoscope 1992;102:501-8.

11. Isaacson GI, Kirchner JC, Kim JH, Kirchner JA. Histology of Isshiki thyroplasty type I. Ann Otol Rhinol Laryngol 1990;99:42-5.

12. Koufman JA, Isaacson G. Laryngoplastic phonosurgery. Otolaryngol Clin North Am 1991;24:1151-77.

\section{Discussion}

Dr. F. Griffith Pearson (Toronto, Ontario, Canada). I do not think many really appreciate the potential importance for thoracic surgeons of the message you are giving. I 
believe this is an important, critical evaluation that you have made of the disability that follows UVCP in patients that thoracic surgeons operate on for malignant disease, particularly lung cancer. It is certainly true that most of them are older, and older patients tolerate these complications less well. I would like to emphasize that I think the major early problem (and you identify 11 patients in whom this procedure was done within 2 weeks of operation) in these patients is cough failure. Nobody worries about hoarseness at this early stage. A few patients aspirate, which makes the cough problem worse. Once you see a few of these palsies, it is easy to recognize the problem the day you first see the patient after operation. This patient has a recurrent nerve palsy, cannot hold air, and cannot raise secretions. I believe that early intervention in selected patients may be very useful.

I note that your data go back to 1991. I have talked with Dr. Ginsberg, and I know that this approach was not particularly used for acute cases at the Memorial Hospital until recently. We have used early augmentation since 1963, when our otolaryngologic staff first started using injectable Teflon. We use glycerin rather than Gelfoam as an interim measure, particularly when one does not know whether paralysis is permanent or transient. If you have not divided the nerve and it was functioning before operation and function is lost after operation, you do not want to do something permanent to a nerve that may recover. You have correctly identified the benefits in late reconstruction, with which I think everyone is familiar: there, largely, it is restoring the patient's voice.

The techniques that we have used have been almost exclusively oral injection of Teflon under topical anesthesia. We have had little experience with the transcervical approach described. I know, again from talking with Dr. Ginsberg, who is familiar with our results in Toronto, that he finds your result a little more precise. He is very impressed with what you have been able to achieve with that approach.

The complications you have are few. The one tracheostomy, however, is alarming. I do not recall our having to do a tracheostomy in any of our patients; certainly in no patient managed early on.

I really wish to emphasize that I think there is an important message that may not be widely appreciated by thoracic surgeons. This approach is useful, particularly in the early postoperative period-the first few days, the first few weeks.

I assume, Dr. Ali, that you are an otolaryngologist. How do you recommend we handle the problem of someone who, at a month or two after resection where the surgeon identified the nerve, thinks it is anatomically intact, but there is still a palsy by clinical examination of the larynx? You note that electromyography may be helpful. Is it absolutely definitive? How do you recommend we proceed with such a patient? At what stage, in other words, is it reasonable to assume that it is a permanent problem and decide to do a permanent-type Teflon injection or the operation you do with the preformed silicone elastomer?

Dr. Ali. As you said, laryngeal electromyography actually is an excellent method of defining the presence of UVCP. I would ask, however, whether the patient still has symptoms, because sometimes if the patient's other cord has compensated appropriately there are no symptoms. If there are still symptoms and the electromyogram, which basically has an excellent role in distinguishing between permanent and temporary paralysis, shows damage, I think at that point we should go ahead with the procedure.

Here I should add that we abandoned the Teflon injections later during the study because it is a permanent procedure. We turned to either Gelfoam or, even better, silicone elastomer because although it sounds like a surgical procedure it is a temporary procedure that can be reversed at any time if the patient's cord function returns. We therefore have nothing to lose, even if we err in our judgment of the permanence of UVCP, in operating on the patient and providing better swallowing and voice. At that point, once we have done all the studies and it is 1 to 2 months after the operation, we should go ahead and perform the procedure. It can always be changed back or temporized.

Dr. Pearson. I am still a little confused. How long would you wait before judging that it is an irreversible palsy? It is a long distance from the aortic arch to the vocal cord, so I assume you have to wait 6 months or so. I would like your expert opinion about that.

Dr. Ali. We do not actually have to wait 6 months for determination of permanence. We waited with some of these patients because that is how they came to us. We can do the procedure earlier if our studies show that it is a permanent paralysis.

Dr. Kraus. There are several issues that I would like to clarify. Laryngeal electromyography, when it does in fact show denervation potentials, is highly specific. In that instance, one can anticipate that the cord function will not return. We are currently in the process of adding this technology to our vocal laboratory.

We individually determine on which patients we operate, and I am unable to provide a uniform approach to every patient. The most significant variable depends on the degree of dysfunction that the patient exhibits. In a hoarse patient who is otherwise free of symptoms, in whom my thoracic colleagues have preserved the neural innervation to the larynx and we are uncertain whether there will be a return in function, I delay surgical intervention for 3 to 6 months. Other factors that impact on this decision are patient issues; early intervention has been used in a number of patients for whom voice is essential to their profession and it is necessary for them to return to their livelihood. As Dr. Ali has correctly pointed out, this is a reversible procedure. It is important to note, however, that in the 75 patients in whom I have performed silicone elastomer medialization, there has been no instance in which I needed to remove a silicone elastomer implant as a consequence of improper assessment of the patient.

Another point that I would like to address is the difference between Teflon and silicone elastomer. I consider myself fortunate to have trained at the Cleveland Clinic with Dr. Harvey Tucker while he was one of the pioneers who introduced this procedure in this country, having adopted it from Isshiki and colleagues, who initially developed it in Japan almost 20 to 25 years ago. Similarly, I was fortunate to obtain a faculty position in an institution that has a highly aggressive group of thoracic 
surgeons who manage a large number of intrathoracic malignancies.

To me, there is no question at this time that Teflon is the least desirable choice. The silicone elastomer procedure is extremely precise. We are no longer dependent on the forces of nature to deposit the Tefion at the desired site. We are able to craft the implant in a fashion that individualizes it to each person's defect. The procedure is performed with local anesthesia, and on the basis of intraoperative measurements, we are able to carve the implant according to each patient's defect. A patient who has a large gap or is a large individual will have a larger implant than will a smaller-bodied patient or an individual with a relatively small gap between the vocal cords.

The final point that I would like to make is that silicone elastomer medialization is an easily taught procedure. I now have three or four fellows who completed our training program and are performing this technique in their practice. It is much easier to teach silicone elastomer medialization than the corresponding Teflon injection technique. Teflon requires the operating surgeon to determine whether the assistant has properly place the needle in the vocal cord and whether the correct amount of Teflon has been adjusted. This is all performed through the narrow confines of a laryngoscope. The open technique of silicone elastomer allows placement of the implant under direct vision, with modification of the implant should it be either too small or too large. For me, of all the permanent techniques this is the procedure of choice.

\section{1-800-55-MOSBY}

This number links you to the full text of articles published in over 25,000 journals, including all Mosby journals. MOSBY Document Express $^{\circledast}$, a rapid response information retrieval service, provides quick turnaround, 24-hour availability, and speedy delivery methods. For inquiries and pricing information, call our toll-free, 24-hour order line: 1-800-55-MOSBY; outside the United States: 415-259-5046; fax: 415-259-5019; E-mail: mosbyexp@class.org.

MOSBY Document Express ${ }^{\text {as }}$ is offered in cooperation with Dynamic Information Corp. 